\title{
CT-Guided Coeliac Plexus Neurolysis in Patients with Intra-Abdominal Malignancy: A Retrospective Evaluation of 52 Palliative In-Patients
}

\author{
Stefan Neuwersch-Sommeregger (D) · Markus Köstenberger • \\ Haro Stettner · Wofgang Pipam • Christian Breschan • \\ Georg Feigl · Rudolf Likar · Markus Egger
}

Received: July 29, 2021 / Accepted: September 2, 2021 / Published online: September 21, 2021

(C) The Author(s) 2021

\begin{abstract}
Introduction: Prevalence rates of chronic abdominal pain (CAP) and breakthrough cancer pain (BTcP) are high in patients with intra-abdominal malignancies. As part of a multimodal approach, CT-guided coeliac plexus blockade (CPB) and CT-guided coeliac plexus neurolysis (CPN) are commonly used pain management strategies. The aim of this study was to evaluate pain outcomes among patients with intra-abdominal malignancies who underwent $\mathrm{CPB}$ and/or CPN.
\end{abstract}

Methods: Patients with intra-abdominal malignancies who underwent $\mathrm{CPB}$ and/or $\mathrm{CPN}$ for

S. Neuwersch-Sommeregger $(\bowtie)$.

M. Köstenberger · W. Pipam · C. Breschan .

R. Likar · M. Egger

Center for Interdisciplinary Pain Therapy, Oncology

and Palliative Care, Klinikum Klagenfurt am

Wörthersee, Feschnigstrasse 11, 9020 Klagenfurt am

Wörthersee, Austria

e-mail: stefan.neuwersch@kabeg.at

S. Neuwersch-Sommeregger · M. Köstenberger ·

C. Breschan

Medical University of Graz, Graz, Austria

H. Stettner

Department of Statistics, Alpen-Adria University

Klagenfurt, Klagenfurt am Wörthersee, Austria

G. Feigl

Institute for Anatomy and Clinical Morphology,

Witten/Herdecke University, Witten, Germany pain control at the general hospital Klagenfurt am Wörthersee from 2010 to 2019 were enrolled. Results: A total of 84 procedures (24 CPB and $60 \mathrm{CPN}$ ) were performed on 52 patients; $62 \%$ of these patients had pancreatic cancer. CPN led to significant pain reduction and decreased BTcP intensity. Patients receiving repeated CPN showed higher individual pain reduction. Higher pre-procedural pain intensity was correlated with higher pain reduction. No difference in pain reduction in patients receiving a diagnostic $\mathrm{CPB}$ prior to $\mathrm{CPN}$ compared to patients without a diagnostic CPB was found. Higher pain reduction after CPN led to longerlasting pain relief. The time frame from diagnosis to CPN was $472( \pm 416)$ days. Patients experienced a mean duration of pain prior to CPN of $330( \pm 53)$ days. The time frame from diagnosis to CPN was shorter in patients with pancreatic cancer compared to other intra-abdominal malignancies. In $58 \%$ of patients pain medication was stable or was reduced after CPN; $16 \%$ of patients complained about pain during the procedure; no major complications occurred. There was no correlation between median survival after CPN and pain outcomes.

Conclusions: In patients with intra-abdominal malignancy-related CAP, CPN is a safe and effective procedure which can provide longlasting significant relief of background pain and BTcP. As part of a multimodal approach, CPN should be considered as an earlier option for pain management in these patients. 


\section{PLAIN LANGUAGE SUMMARY}

In patients with intra-abdominal malignancyrelated abdominal pain, CT-guided coeliac plexus neurolysis is a safe and effective procedure. It can provide long-lasting significant relief of background pain and breakthrough cancer pain. As part of a multimodal approach, coeliac plexus neurolysis should be considered as an earlier option for pain management in patients with intractable abdominal cancer pain.

Keywords: CT-guided coeliac plexus neurolysis; Chronic abdominal pain; Cancer pain; Intra-abdominal malignancy

\section{Key Summary Points}

Why carry out this study?

In patients with intra-abdominal malignancies, chronic abdominal pain (CAP) has a prevalence up to $50 \%$, and has a huge impact on patients' quality of life and disability.

Patients with uncontrolled CAP due to intra-abdominal malignancies develop breakthrough cancer pain (BTcP) in up to $70 \%$ of cases.

The primary objective of this study was to evaluate pain outcomes among patients with intra-abdominal malignancies who underwent CT-guided coeliac plexus neurolysis (CPN).

\section{What was learned from the study?}

In patients with intra-abdominal malignancy-related abdominal pain, CPN is a safe and effective procedure which can provide long-lasting significant relief of background pain and BTcP.

As part of a multimodal approach, CPN should be considered as an earlier option for pain management in patients with intractable abdominal cancer pain.

\section{INTRODUCTION}

Chronic abdominal pain (CAP) has a prevalence up to $50 \%$ in patients with intra-abdominal malignancies, and has a huge impact on patients' quality of life and disability [3, 24]. Obstruction of hollow viscus, like biliary tract or intestine, or infiltration by cancer masses of the abdominal wall, retroperitoneal, or pelvic structures causes visceral pain due to mixed neuropathic and nociceptive mechanisms [19]. Patients with uncontrolled CAP due to intraabdominal malignancies develop breakthrough cancer pain (BTCP) in up to $70 \%$ of cases [3]. Treatment options as part of a multimodal approach, and standards for the management of cancer-related pain [1], range from medical treatment, typically opioids and centrally targeted pharmacotherapy, as well as interventional pain management procedures [24]. Despite the commonly known side effects of opioids, such as constipation, sedation, dependence, nausea, and respiratory depression or spasm of the sphincter of Oddi, CAP is in many cases refractory to opioid therapy, leading to a vicious circle of dose escalation and unwanted drug side effects $[17,27]$. Thus, percutaneous interventional techniques like CT-guided coeliac plexus blockade (CPB) or CT-guided coeliac plexus neurolysis (CPN) are commonly used pain management strategies to decrease pain, increase quality of life, and reduce opioid therapy $[24,30]$.

The coeliac plexus is the largest autonomic plexus, composed of nerve fibers and ganglia, and because of its radial shape it is known as the solar plexus. The coeliac plexus is located over the anterior and lateral surface of the aorta, next to the origin of the coeliac trunk and the superior mesenteric and renal arteries [8]. It is located in the retroperitoneal fat, posterior to the pancreas and stomach, and separated posteriorly by the diaphragmatic crura [24]. The following nerves join the coeliac plexus: (1) preganglionic sympathetic efferent nerve fibers from the lower thoracic and upper lumbar ganglia; (2) the end of the posterior vagal trunk of the esophageal plexus; (3) the greater and lesser splanchnic nerve [8]. The coeliac plexus 
comprises two big paired and many smaller ganglia: right and left from the origin of the coeliac trunk from the aorta, a left and right coeliac ganglion is located. More caudally beneath the origin of the superior mesenteric artery, the superior mesenteric ganglion is located. Next to the renal artery, the renal ganglia and the aoriticorenal ganglia are found $[8,24]$.

Preganglionic parasympathetic efferent fibers from the posterior vagal trunk are also found in the coeliac plexus. These fibers carry autonomic supply to the pancreas, liver, gallbladder, stomach, spleen, kidneys and adrenal glands, small and large bowel, and abdominal blood vessels [2, 24]. Along with splanchnic nerves, also visceral afferent fibers that provide nociceptive stimuli from distal esophagus to the colon pass through the coeliac plexus terminating in the spinal cord [21, 24]. Nociceptive signals then pass through the spinal cord to the thalamus and cortex. As a result of its pain transmission the coeliac plexus is a veritable target for controlling pain from upper abdominal organs $[13,24]$.

Compared to $\mathrm{CPB}$ with injection of corticosteroids and/or long-acting local anesthetics, $\mathrm{CPN}$ leads to permanent destruction of the coeliac plexus after injection of phenol or ethanol, due to protein coagulation and necrosis of neural structures [12, 13, 24]. CPB is commonly practiced prior to $\mathrm{CPN}$ as a diagnostic blockade, although its clinical significance in clinical routine is questionable [29].

CPN may be accomplished using an anterior para-aortic [13, 22], a bilateral posterior paraaortic [2, 24], a posterior transaortic [13, 15], or a trans-intervertebral disc approach $[9,24]$. In all of these approaches, using multidetector computed tomography guidance, patient anatomy, tumor masses, and the needle tip are clearly visible, and hence complication rates are low $[13,20,24]$. The most typical side effect is back pain, which mostly radiates to the shoulder, resulting from neurolysis of sensory nerve fibers [13]. Other side effects are transient pain at the injection site, or diarrhea and hypotension, due to sympathetic blockade [2]. Serious side events, like bleeding complications [26], retroperitoneal hematoma [20], lower extremity paralysis [23], thrombosis of the coeliac trunk or vasospasm of the coeliac artery leading to hepatic, splenic, gastric, or bowel infarction [7, 24] are rare. Complications due to poor needle placement, e.g., kidney injuries, pneumothorax, or neurologic complications due to inadvertent injection of neurolytic agent are scarce when CT guidance is used [13].

The primary objective of this study was to evaluate pain outcomes among patients with intra-abdominal malignancies who underwent $\mathrm{CPN}$. We further analyzed indications, details of the procedure, pre- and post-procedural pain intensities and $\mathrm{BTCP}$, and changes in pain medication after the procedure. Such information should lead to better implementation of CPN in a multimodal approach of pain management and thus improve health care quality in patient with intra-abdominal malignancies.

\section{METHODS}

\section{Ethics}

This retrospective study was approved by the Ethical Committee of Carinthia (S2020-14, 18 April 2020), conducted according to the Helsinki declaration and International Association for the Study of Pain (IASP)'s guidelines for pain research in animals and humans, and authorized by the hospital general management. The requirement for written informed consent was waived by the ethics committee.

\section{Setting}

All patients with intra-abdominal malignancies who underwent CPB and/or CPN for pain control at the general hospital Klagenfurt am Wörthersee between January 2010 and January 2019 were identified using our clinical data retrieval system and procedure notes. Each case was reviewed as to abstract baseline demographic data and clinical characteristics, as well as pain- and procedure-related data. Demographic and clinical data were gender, age, primary diagnosis, diagnosis date, and location of the malignancy. 
Procedure-related data included details of the procedural approach and injectate, complications, time frame from diagnosis to $\mathrm{CPB} / \mathrm{CPN}$, and time frame from start of pain to $\mathrm{CPB} / \mathrm{CPN}$. Pain-related data were pre- and post-procedural pain scores (baseline and pain peaks), pre- and post-procedural pain medication, and duration of CPN success. The efficacy of pain control was evaluated before and 1 day after $\mathrm{CPB}$ on the basis of the numeric rating scale (11-item NRS; 0 , "no pain"-10, "worst pain imaginable"). Patients with incorrect medical records or ambiguous documentation and outcome measures were excluded.

\section{Statistical Analysis}

Descriptive statistics were used for the characterization of the observed variables. Appropriate parametric or non-parametric tests were done. Variables were compared by $U$ test (Wilcoxon-Mann-Whitney test) and Kruskal-Wallis test, and by $t$ test and one-factor analysis of variance (ANOVA) in case of normally distributed data. Deviation from zero was investigated by the signed rank test, and by the onesample $t$ test in case of normally distributed data. Significance of trends was tested by the Jonckheere test. The Kolmogorov-Smirnov test was used for proving normality. Spearman rank correlation coefficient and Pearson correlation coefficient were calculated to examine association between variables. Associations in cross-tables were investigated by chi-square test, and in case of $2 \times 2$ tables also by exact Fisher test. Significance was defined as a $p$ value less than $0.05 ; p$ values are reported to a maximum of three decimal places. Differences in median survival were investigated using the Kaplan-Meier method and compared using a log-rank test. Cox proportional hazard model was used to examine association between variables. For all calculations the statistical package $\mathrm{R}$ version 4.0.3 and Hewlett-Packard RPL version 2.15 were used.

\section{Diagnostic CPB and CPN}

One physician, who is an expert in this field with 25 years of experience in this technique, performed all CPB and CPN using an anterior approach $[13,24]$ according to a standardized protocol and technique. No premedication and no sedation during the procedure was given to prevent potential hypotensive events. Patients were positioned in a supine position and local infiltration with $2 \%$ lignocaine was used. A needle $\quad$ (Sterican ${ }^{\circledR}$ G23 × $31 / 8^{\prime \prime}$; $\varnothing$ $0.60 \times 80 \mathrm{~mm}$, B. Braun Austria Ges.m.b.H.) was inserted through the anterior abdominal wall into the retropancreatic space which correspondents to the retroperitoneal space [25] and the needle tip was placed anterior to the aorta and the diaphragmatic crura between the superior mesenteric artery and the roots of the coeliac plexus [13]. After confirmation of the needle tip with nonionic contrast medium (Jopamidol/Jopamiro ${ }^{\circledR}$ ), $10 \mathrm{ml}$ of bupivacain $0.25 \%$ (Bucain $^{\circledR} 0.25 \%$ ) in $\mathrm{CPB}$ and $10 \mathrm{ml}$ of ethanol $95 \%$ in CPN were injected into the antecrural space.

\section{RESULTS}

\section{Demographic Data}

Thirty-six percent of patients were female, and $64 \%$ were male. The mean age was 65 years, with a range from 24 to 88 years with normal distribution. Baseline demographic and main results are shown in Table 1.

\section{CPB and CPN}

In summary, 84 procedures $(24 \mathrm{CPB}$ and 60 CPN) were performed on 52 individual patients. Five patients (9\%) had a single diagnostic CPB and 19 patients $(36.5 \%)$ had diagnostic $\mathrm{CPB}$ prior to CPN. Thirty-five patients (64\%) had one CPN, 11 patients (20\%) had two CPN, and 1 patient $(2 \%)$ had $3 \mathrm{CPN}$.

\section{Diagnosis}

Table 1 shows the primary malignancy of patients who underwent the procedure. All patients were diagnosed with intra-abdominal malignancies and $62 \%$ had pancreatic cancer. 
Table 1 Demographic data, results

\begin{tabular}{|c|c|}
\hline \multicolumn{2}{|l|}{ Age; years } \\
\hline Mean (SD) & $64.9(13.6)$ \\
\hline Min-max & 24-88 \\
\hline \multicolumn{2}{|l|}{ Sex; $n(\%)$} \\
\hline Female & $20(36)$ \\
\hline Male & $35(64)$ \\
\hline \multicolumn{2}{|l|}{ Primary malignancy; $n(\%)$} \\
\hline Pancreas & $34(61.8)$ \\
\hline Stomach & $4(7.3)$ \\
\hline Esophagus & $4(7.3)$ \\
\hline Gall bladder & $5(9.1)$ \\
\hline Other & $8(14.5)$ \\
\hline \multicolumn{2}{|l|}{ Mean pain intensity; NRS (SD) } \\
\hline Before 1st CPN & $5.1(1.9)$ \\
\hline After 1st CPN & $2.4(2.0)$ \\
\hline \multicolumn{2}{|l|}{ Mean pain intensity; NRS (SD) } \\
\hline Before 2nd CPN & $5.5(1.3)$ \\
\hline After 2nd CPN & $2.0(1.0)$ \\
\hline \multicolumn{2}{|l|}{ BTcP; NRS (SD) } \\
\hline Before CPN & $7.8(1.1)$ \\
\hline After CPN & $4.8(3.0)$ \\
\hline \multicolumn{2}{|l|}{ Duration of pain reduction; days (SD) } \\
\hline 1 st $\mathrm{CPN}$ & $78(96)$ \\
\hline 2nd $\mathrm{CPN}$ & $120(109)$ \\
\hline \multicolumn{2}{|l|}{ Time frame } \\
\hline Diagnosis to CPN, days (SD) & $472(416)$ \\
\hline Onset of pain to CPN, days (SD) & $330(53)$ \\
\hline \multicolumn{2}{|l|}{ Complications (\%) } \\
\hline Pain during procedure & 16 \\
\hline \multicolumn{2}{|l|}{ Pain medication after CPN (\%) } \\
\hline Stable or reduced & 58 \\
\hline Increased & 42 \\
\hline
\end{tabular}

Table 1 continued

Median survival after diagnosis; days (95\% CI)

All patients $(n=46)$

$342(233-452)$

Pancreatic cancer $(n=28)$

$280(213-347)$

Other $(n=18)$ $480(477-487)$

Median survival after 1 st CPN; days (95\% CI)

All patients $(n=46)$

$64(57-71)$

Pancreatic cancer $(n=28)$

$72(30-114)$

Other $(n=18)$ $53(40-66)$

Median survival after 2nd CPN; days (95\% CI)

$$
\begin{array}{ll}
\text { All patients }(n=46) & 40 \text { (n.a.) } \\
\text { Pancreatic cancer }(n=28) & 62 \text { (n.a.) } \\
\text { Other }(n=18) & 37(19-55)
\end{array}
$$

n.a.: not available

\section{Pain Intensity}

Before the first $\mathrm{CPN}$, the mean pain intensity (11-item NRS; 0, "no pain"-10, "worst pain imaginable") was $5.1( \pm 1.9)$, with a statistically significant $(p<0.01)$ pain reduction after the first CPN $(2.4 \pm 2.0)$ (see Fig. 1).

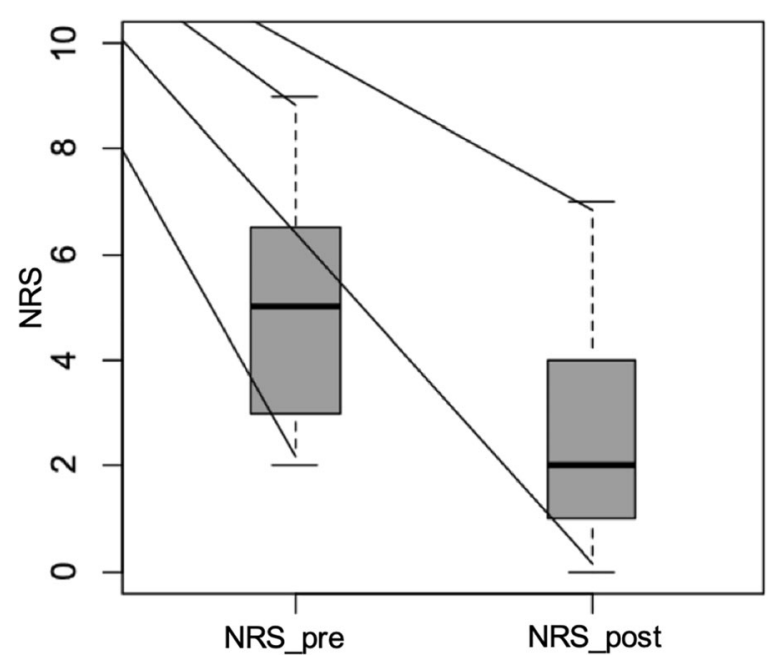

Fig. 1 Boxplot of NRS before and after first CPN 
Before the second $\mathrm{CPN}$, the mean pain intensity was $5.5( \pm 1.3)$, with a statistically significant $(p<0.01)$ pain reduction after the procedure $(2.0 \pm 1.0)$ (see Fig. 2 ).

We found no statistically significant difference $(p=0.2)$ in mean pain reduction after the first and second CPN, but a statistically significant $(p=0.025)$ correlation of pain reduction and number of CPN was shown. Furthermore, a higher pre-procedural pain intensity was highly significant $(p<0.01)$ correlated with a higher pain reduction after blockade. There was no statistically significant difference $(p=0.43)$ in pain reduction in patients receiving a diagnostic CPB prior to first and second CPN compared to patients without a diagnostic CPB.

There was no difference $(p=0.36)$ in pain reduction after $\mathrm{CPN}$ depending on the primary diagnosis.

\section{BTcP}

Before CPN the intensity of BTcP was $7.8( \pm 1.1)$ with a statistically significant $(p<0.01)$ reduction of intensity after the CPN $(4.8 \pm 3.0)$ (see Fig. 3).

There was no difference of reduction of BTcP depending on the number of blockades, pain intensity prior to procedure, or diagnosis.

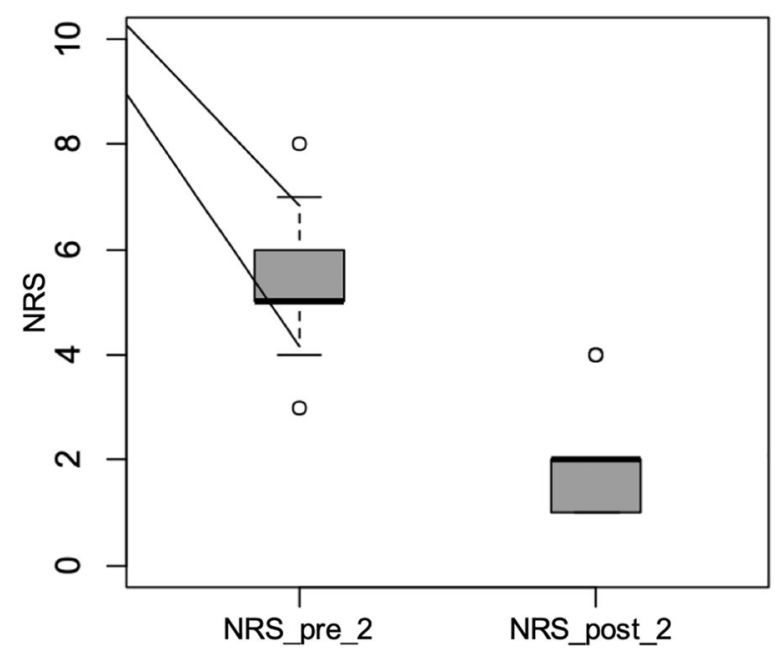

Fig. 2 Boxplot of NRS before and after second CPN

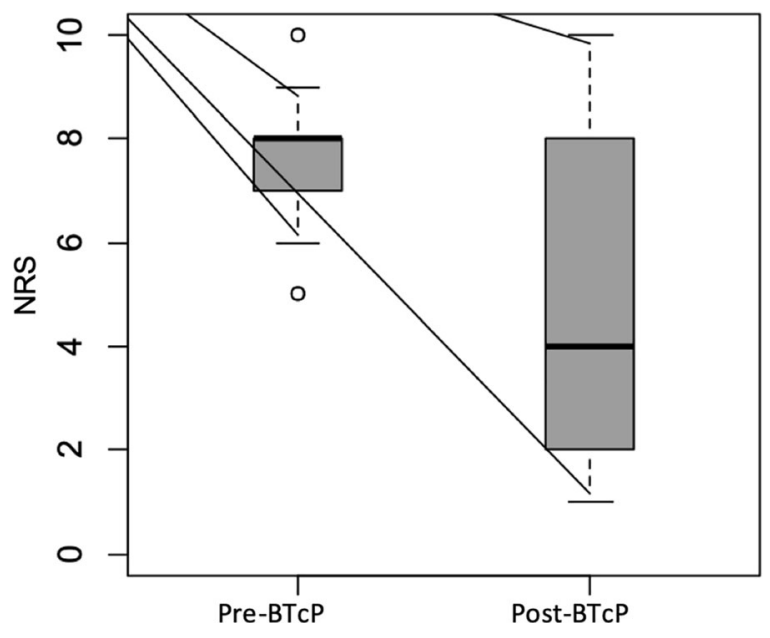

Fig. 3 Boxplot of NRS of BTcP before and after CPN

\section{Duration of Pain Reduction}

The mean duration of pain reduction after the first CPN was $78( \pm 96)$ days, and $120( \pm 109)$ days after the second CPN. The median difference (88 vs. 53) of the duration of pain reduction after the first and second CPN was nearly statistically significant $(p=0.101)$.

A significant $(p=0.048)$ correlation of pain reduction and duration of pain reduction was found: higher pain reduction after CPN leads to longer-lasting pain reduction.

Patients with pancreatic cancer showed a slightly $(p=0.081)$ longer reduction of pain intensity compared to patients with other intraabdominal malignancies. Patients with pancreatic cancer did not receive more CPN compared with other intra-abdominal malignancies.

\section{Time Frame}

The time frame from diagnosis to CPN was 472 $( \pm 416)$ days, and patients experienced a mean duration of pain prior to CPN of $330( \pm 53)$ days. It has been shown that the time frame from diagnosis to CPN was significantly $(p<0.01)$ shorter in patients with pancreatic cancer compared to other intra-abdominal malignancies. The mean time frame from diagnosis to CPN in patients with pancreatic cancer was $175( \pm 175)$ days. 


\section{Medication}

Patients were divided into three groups depending on their medication after CPN: group 1, increase of pain medication; group 2, decrease of pain medication; group 3, no change in pain medication.

In $58 \%$ of patients pain medication was stable or was reduced after CPN. Group 1 showed significant higher $(p=0.038)$ post-procedural pain scores compared to groups 2 and 3 .

There was no correlation between change in pain medication and number of CPN, reduction of pain intensity, and primary diagnosis, respectively.

\section{Complications}

Sixteen percent of patients complained about pain during the procedure. No major complications occurred in patients who underwent CPN.

\section{Survival}

In our study population, the median survival from the date of diagnosis was 342 days (95\% CI 233-452). The median survival from the date of first CPN was 64 days (95\% CI 57-71) and from the date of second CPN was 40 days (95\% CI, n.a.) (see Table 1). Kaplan-Meier curves for the survival of patients with pancreatic cancer and those with other cancers are illustrated in Fig. 4. The median survival was significantly lower $(p<0.01)$ for patients with pancreatic cancer compared to combined other cancer entities. There was no correlation between median survival after $\mathrm{CPN}$ and respectively pain reduction after first and second CPN, reduction of BTcP, change in pain medication, time frame from diagnosis to $\mathrm{CPN}$, or duration of pain reduction after first or second CPN.

\section{DISCUSSION}

This present study describes pain outcomes among patients with intra-abdominal malignancies who underwent CPN. Despite multimodal pain management strategies in these patients, CAP and BTcP are still common and have a major impact on the patient's disability and quality of life $[3,17,24]$. In this context, CPN is a palliative intervention with the aim to reduce intractable pain and narcotic requirements [4].

In an earlier evaluation of all in-patients in the general hospital of Klagenfurt am Wörthersee, a mean overall current pain intensity at rest of 1.9 ( \pm 2.5$)$ (11-item NRS) was found [11]. In the present setting, the mean pain intensity was $5.1( \pm 1.9)$ before first CPN and $5.5( \pm 1.3)$ before second CPN with BTcP episodes of 7.8 $( \pm 1.1)$. Most of these patients were diagnosed with pancreatic cancer. Not only does this cancer entity have high mortality rates and poor prognosis [14] but the predominant symptom is abdominal discomfort and pain at the time of diagnosis [28]. Adequate pain

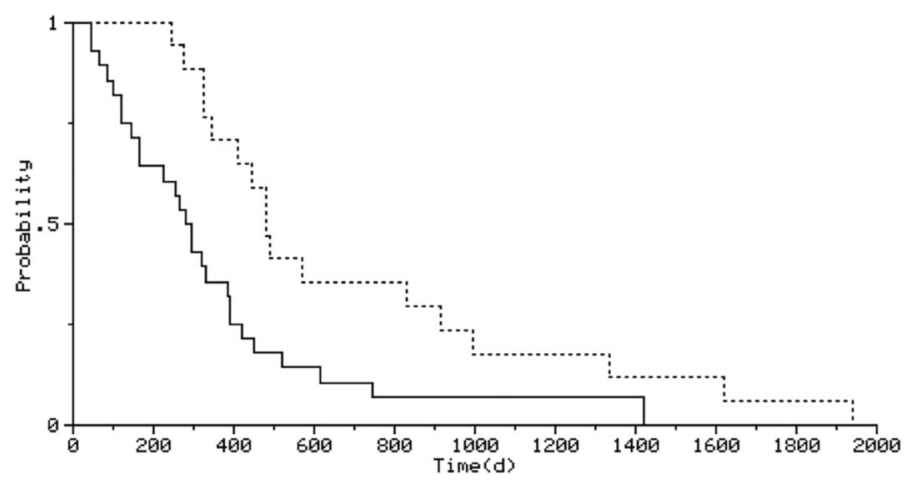

Fig. 4 Kaplan-Meier curves for survival between patients with pancreatic cancer (continuous line) and other cancer entities (dotted line) after CPN $(\log$-rank $p<0.01)$ 
control is crucial given that high background pain intensity and episodes of BTcP negatively affect the patient's mobilization, mood, and sleep [11] and that many patients with pancreatic cancer or other intra-abdominal malignancies have advanced or inoperable disease at the time of diagnosis $[14,28]$.

After the first and second CPN we showed major pain relief of current pain, which was independent of the intra-abdominal malignancy. This supports previous literature that showed CPN as an effective procedure for the management of CAP $[4,5,24]$. Moreover, a reduction was found in the intensity of $\mathrm{BTCP}$, independent of the number of procedures or pain intensity prior to the procedure. Interestingly, patients receiving two CPN showed higher individual pain reduction. This stands in contrast to McGreevy et al. [18], who showed that repeated coeliac plexus neurolysis (rCPN) does not lead to better pain relief as the initial CPN.

One factor that was strongly associated with good analgesia after CPN was high pre-procedural pain intensity. Higher pre-procedural pain intensities led to higher pain reduction after the intervention, which stands in contrast to earlier studies $[6,28]$ showing that a low daily opioid dose and good performance status before CPN was clearly associated with good CPN outcome. The authors concluded that higher opioid doses could reflect poorly controlled pain associated with a more significant tumor burden and recommended CPN as an early option for pain management before opioid dose escalation $[6,28]$.

No difference was found in pain reduction, side effects, or complications in patients receiving a diagnostic block prior to the first or second CPN compared to patients without a diagnostic block. These results underline the results of Yuen et al. [29], who stated that the clinical significance of $\mathrm{CPB}$ is questionable and that its routine use is not warranted before CPN. Ischia et al. [10] showed that a CPB may be false positive for different reasons: a placebo effect, differences in local spread of neurolytic agents and local anesthetics, systemic absorption of the anesthetic. Moreover, a prior CPB may lead to higher procedure-related risks, higher health care costs, or possible false negative results $[10,29]$.

It has been shown that CPN leads to longlasting pain control in up to $90 \%$ of patients with different intra-abdominal malignancies, particularly in patients with pancreatic cancer [5]. The results of our study support this finding, as the mean duration of pain reduction after the first CPN was $78( \pm 96)$ days, and $120( \pm 109)$ days after the second CPN, and patients with pancreatic cancer showed a trend to longerlasting pain reduction compared to other intraabdominal malignancies. Interestingly, higher pain reduction after CPN led to longer-lasting pain control.

It has also been stated that successful CPN reduces analgesic consumption and hence reduces the incidence of unwanted drug side effects [13]. In our survey, 58\% of our patients' pain medication was stable or reduced after successful CPN. These patients also showed significantly lower pain scores compared to patients with the necessity to increase analgesic consumption after the procedure.

Unfortunately, pain medication had to be increased in $42 \%$ of our patients. This shows the importance of timing of CPN: patients with poor pre-procedural performance status are more likely to show higher levels of pain [16], and patients with advanced cancer may show multisite mixed pain patterns [28], which may lead to poor analgesic outcomes. Approximately 1 year after onset of pain, patients with intraabdominal malignancies received CPN. Surprisingly, patients with diagnosed pancreatic cancer wait half as long for this procedure. Taking into account that it has been shown that $\mathrm{CPN}$ is a safe and effective procedure, our results suggest that CPN should be considered as an earlier option for pain management in these patients.

Yoon et al. [28] showed that good performance status is an independent predictor for good analgesic efficacy following $\mathrm{CPN}$ in patients with pancreatic cancer and that patients with good analgesia after CPN tended to have shorter pain duration before the procedure. Furthermore, they found that the median survival time after CPN for patients with good analgesia from CPN was significantly 
longer than that of patients with poor pain relief. They also showed that patients with poor analgesia after CPN already had an advanced stage of cancer and poor performance status. In this current study, the median survival time after CPN was not associated with pain reduction after first and second $\mathrm{CPN}$, reduction of $\mathrm{BTCP}$, change in pain medication after $\mathrm{CPN}$, time frame from diagnosis to $\mathrm{CPN}$, or duration of pain reduction after first or second CPN, neither in patients with pancreatic cancer nor in patients with other cancer entities. Even though this result stands in contrast to Yoon et al. [28], we acknowledge that survival outcomes do seem to be affected more by the patients' conditions as well as the progression of the disease, and less so than the analgesic effect of CPN. Nevertheless, we strongly recommend an early CPN in selected patients.

There are several important limitations in our study that deserve mention. One is that it used a retrospective design in a single center. Therefore, we could not completely evaluate detailed pain-related outcomes after discharge. In addition, given the poor life expectancy of most of our patients, long-term follow-up was not possible. As already described in earlier studies [28], this survey used a real-world clinical practice model in which different pain physicians planned the treatment approach for managing cancer pain. So, the decision for performing a CPN or other treatment options could have been influenced by their own preconceptions, and may bias our study population. Despite these limitations, we feel that our large study population, our standardized procedure, and the fact that the technique was performed by one pain physician only allow us to make valid conclusions.

\section{CONCLUSIONS}

$\mathrm{CPN}$ is a safe and effective procedure in patients with intra-abdominal malignancy-related abdominal pain and can provide long-lasting significant relief of background pain and BTcP. As part of a multimodal approach, CPN should be considered as an earlier option for pain management in patients with intractable abdominal cancer pain.

\section{ACKNOWLEDGEMENTS}

Funding. No funding or sponsorship was received for this study or publication of this article. The journal's Rapid Service Fee was funded by the authors.

Authorship. All named authors meet the International Committee of Medical Journal Editors (ICMJE) criteria for authorship for this article, take responsibility for the integrity of the work as a whole, and have given their approval for this version to be published.

Author Contributions. Stefan NeuwerschSommeregger: substantial contribution to conception and design, interpretation of data, drafting, final approval of the final version.Markus Köstenberger: substantial contribution to conception and design, interpretation of data, drafting, final approval of the final version. Haro Stettner: statistical analysis, interpretation of data.Wolfgang Pipam: statistical analysis, interpretation of data. Christian Breschan: revising the article and interpretation of data.Georg Feigl: interpretation of data, drafting, and revising the article.Rudolf Likar: substantial contribution to conception and design, interpretation of data, drafting, final approval of the final version.Markus Egger: substantial contribution to conception and design, interpretation of data, drafting, final approval of the final version.

Disclosures. Stefan Neuwersch-Sommeregger, Markus Köstenberger, Haro Stettner, Wofgang Pipam, Christian Breschan, Georg Feigl, Rudolf Likar, and Markus Egger confirm that no conflicts of interest exist.

Compliance with Ethics Guidelines. This retrospective study was approved by the Ethical Committee of Carinthia (S2020-14, 18 April 2020), conducted according to the Helsinki declaration and IASP's guidelines for pain 
research in animals and humans, and authorized by the hospital general management. The requirement for written informed consent was waived by the ethics committee.

Data Availability. The datasets generated during and/or analyzed during the current study are available from the corresponding author on reasonable request.

Open Access. This article is licensed under a Creative Commons Attribution-NonCommercial 4.0 International License, which permits any non-commercial use, sharing, adaptation, distribution and reproduction in any medium or format, as long as you give appropriate credit to the original author(s) and the source, provide a link to the Creative Commons licence, and indicate if changes were made. The images or other third party material in this article are included in the article's Creative Commons licence, unless indicated otherwise in a credit line to the material. If material is not included in the article's Creative Commons licence and your intended use is not permitted by statutory regulation or exceeds the permitted use, you will need to obtain permission directly from the copyright holder. To view a copy of this licence, visit http://creativecommons.org/licenses/by$\mathrm{nc} / 4.0 /$.

\section{REFERENCES}

1. Bennett MI, Eisenberg E, Ahmedzai SH, et al. Standards for the management of cancer-related pain across Europe-A position paper from the EFIC Task Force on Cancer Pain. Eur J Pain. 2019;23:660-8.

2. Cornman-Homonoff J, Holzwanger DJ, Lee KS, et al. Celiac plexus block and neurolysis in the management of chronic upper abdominal pain. Semin Intervent Radiol. 2017;34:376-86.

3. Cuomo A, Cascella M, Forte CA, et al. Careful breakthrough cancer pain treatment through rapidonset transmucosal fentanyl improves the quality of life in cancer patients: results from the BEST multicenter study. J Clin Med. 2020;9:1003.

4. Edelstein MR, Gabriel RT, Elbich JD, et al. Pain outcomes in patients undergoing CT-guided celiac plexus neurolysis for intractable abdominal visceral pain. Am J Hosp Palliat Care. 2017;34:111-4.

5. Eisenberg E, Carr DB, Chalmers TC. Neurolytic celiac plexus block for treatment of cancer pain: a meta-analysis. Anesth Analg. 1995;80:290-5.

6. Erdek MA, Halpert DE, Gonzalez Fernandez M, et al. Assessment of celiac plexus block and neurolysis outcomes and technique in the management of refractory visceral cancer pain. Pain Med. 2010;11: 92-100.

7. Gimeno-Garcia AZ, Elwassief A, Paquin SC, et al. Fatal complication after endoscopic ultrasoundguided celiac plexus neurolysis. Endoscopy. 2012;44(Suppl 2):E267.

8. Hafferl W. Lehrbuch der topographischen Anatomie. Berlin: Springer-Verlag; 1969.

9. Ina $\mathrm{H}$, Kitoh $\mathrm{T}$, Kobayashi $\mathrm{M}$, et al. New technique for the neurolytic celiac plexus block: the transintervertebral disc approach. Anesthesiology. 1996;85:212-7.

10. Ischia S, Ischia A, Polati E, et al. Three posterior percutaneous celiac plexus block techniques. A prospective, randomized study in 61 patients with pancreatic cancer pain. Anesthesiology. 1992;76: $534-40$

11. Jaksch W, Neuwersch S, Reichhalter R, et al. Pain in hospital: assessing the pain situation in Austrian patients. Schmerz. 2015;29:625-31.

12. Jones RR. A technic for injection of the splanchnic nerves with alcohol. Anesth Analg. 1957;36:75-7.

13. Kambadakone A, Thabet A, Gervais DA, et al. CTguided celiac plexus neurolysis: a review of anatomy, indications, technique, and tips for successful treatment. Radiographics. 2011;31:1599-621.

14. Kamisawa T, Wood LD, Itoi $\mathrm{T}$, et al. Pancreatic cancer. Lancet. 2016;388:73-85.

15. Lieberman RP, Waldman SD. Celiac plexus neurolysis with the modified transaortic approach. Radiology. 1990;175:274-6.

16. Lin CC, Lai YL, Ward SE. Effect of cancer pain on performance status, mood states, and level of hope among Taiwanese cancer patients. J Pain Symptom Manag. 2003;25:29-37.

17. Manchikanti L, Manchikanti KN, Kaye AD, et al. Challenges and concerns of persistent opioid use in cancer patients. Expert Rev Anticancer Ther. 2018;18:705-18. 
18. Mcgreevy K, Hurley RW, Erdek MA, et al. The effectiveness of repeat celiac plexus neurolysis for pancreatic cancer: a pilot study. Pain Pract. 2013;13:89-95.

19. Mercadante S, Adile C, Masedu F, et al. Breakthrough cancer pain in patients with abdominal visceral cancer pain. J Pain Symptom Manag. 2019;57:966-70.

20. Moore DC. The dreaded complications from neurolytic celiac plexus blocks are preventable! Reg Anesth Pain Med. 2004;29:377-8.

21. Noble M, Gress FG. Techniques and results of neurolysis for chronic pancreatitis and pancreatic cancer pain. Curr Gastroenterol Rep. 2006;8:99-103.

22. Romanelli DF, Beckmann CF, Heiss FW. Celiac plexus block: efficacy and safety of the anterior approach. AJR Am J Roentgenol. 1993;160:497-500.

23. Seicean A. Celiac plexus neurolysis in pancreatic cancer: the endoscopic ultrasound approach. World J Gastroenterol. 2014;20:110-7.

24. Urits I, Jones MR, Orhurhu V, et al. A comprehensive review of the celiac plexus block for the management of chronic abdominal pain. Curr Pain Headache Rep. 2020;24:42.
25. Wang PJ, Shang MY, Qian Z, et al. CT-guided percutaneous neurolytic celiac plexus block technique. Abdom Imaging. 2006;31:710-8.

26. Warner NS, Moeschler SM, Warner MA, et al. Bleeding complications in patients undergoing celiac plexus block. Reg Anesth Pain Med. 2016;41: 488-93.

27. Wyse JM, Chen YI, Sahai AV. Celiac plexus neurolysis in the management of unresectable pancreatic cancer: when and how? World J Gastroenterol. 2014;20:2186-92.

28. Yoon DM, Yoon KB, Baek IC, et al. Predictors of analgesic efficacy of neurolytic celiac plexus block in patients with unresectable pancreatic cancer: the importance of timing. Support Care Cancer. 2018;26:2023-30.

29. Yuen TS, Ng KF, Tsui SL. Neurolytic celiac plexus block for visceral abdominal malignancy: is prior diagnostic block warranted? Anaesth Intensive Care. 2002;30:442-8.

30. Zhong W, Yu Z, Zeng JX, et al. Celiac plexus block for treatment of pain associated with pancreatic cancer: a meta-analysis. Pain Pract. 2014;14:43-51. 